\title{
The Group Evolution Multiwavelength Study (GEMS): The Sample and Datasets
}

\author{
Duncan A. Forbes ${ }^{\mathrm{A}, \mathrm{F}}$, Trevor Ponman ${ }^{\mathrm{B}}$, Frazer Pearce ${ }^{\mathrm{C}}$, John Osmond ${ }^{\mathrm{D}}$, Virginia \\ Kilborn $^{\mathrm{A}}$, Sarah Brough ${ }^{\mathrm{A}}$, Somak Raychaudhury ${ }^{\mathrm{B}}$, Carole Mundell $^{\mathrm{E}}$, Trevor Miles ${ }^{\mathrm{B}}$, and \\ Katie $\operatorname{Kern}^{\mathrm{A}}$ \\ A Centre for Astrophysics \& Supercomputing, Swinburne University of Technology, Hawthorn VIC 3122 , \\ Australia \\ B School of Physics and Astronomy, University of Birmingham, Birmingham B15 2TT, UK \\ C School of Physics and Astronomy, University of Nottingham, Nottingham NG7 2RD,UK \\ D Centre for Electronic Imaging, School of Engineering and Design, Brunel University, Uxbridge UB8 \\ $3 \mathrm{PH}, \mathrm{UK}$ \\ E Astrophysics Research Institute, Liverpool John Moores University, Birkenhead CH41 1LD, UK \\ F Corresponding author. Email: dforbes@swin.edu.au
}

Received 2005 December 19, accepted 2006 February 7

\begin{abstract}
Galaxy groups have been under-studied relative to their richer counterparts - clusters. The Group Evolution Multiwavelength Study (GEMS) aims to redress some of the balance. Here we describe the GEMS sample selection and resulting sample of 60 nearby $(<130 \mathrm{Mpc})$ galaxy groups and our multiwavelength dataset of X-ray, optical, and Hi imaging. ROSAT X-ray images of each group are presented. GEMS also utilizes near-infrared imaging from the 2MASS survey and optical spectra from the 6dFGS. These observational data are complemented by mock group catalogues generated from the latest $\Lambda \mathrm{CDM}$ simulations with gas physics included. Existing GEMS publications are briefly highlighted as are future publication plans.
\end{abstract}

Keywords: surveys: groups — galaxies: evolution — galaxies: formation — galaxies: groups

\section{Introduction}

The degree to which initial formation and environmental influences determine galaxy properties today is similar to the 'Nature versus Nurture' debate. Like the biological debate, this issue is an ongoing and fundamental one for contemporary astrophysics. In addition to the environment having a role in galaxy evolution, the galaxies themselves also influence their local environment through expelled gas, high-energy particles, and radiation outflows. This may in turn feedback into the subsequent evolution of galaxies within that environment.

Quantifying the importance of this complex interplay between galaxies and their environment requires the detailed tracking of galaxy properties over a broad range of environments and look-back times. In terms of environment, there are three broad regimes based on the spatial density of galaxies - rich clusters, groups, and the low-density 'field'. At the highest densities, rich clusters contain hundreds, even thousands, of galaxies within a few cubic megaparsecs. In contrast, field galaxies are relatively isolated galaxies whose nearest $\mathrm{L}^{*}$ neighbours can be many megaparsecs away. Galaxy groups represent an environment that is intermediate between these two extremes, containing a few tens of galaxies within a cubic megaparsec. Despite the meagre size of their populations, groups are vital to obtaining a complete understanding of galaxy evolution and environmental processes for the following reasons.

- Groups are the most common galaxy environment: Approximately $70 \%$ of galaxies in the local universe are located in groups (Tully 1987).

- Groups are the building blocks of bigger structures: In the hierarchical clustering scenario, groups are the fundamental building blocks of galaxy clusters. Hence the evolution of galaxies in groups is highly relevant to the early evolution of rich cluster galaxies (e.g. Bekki 1999; Solanes et al. 1999; Moss \& Whittle 2000).

- Groups are the most favoured environment for galaxy mergers: The best chance of observing galaxy mergers and interactions is in groups because of the low relative velocities between galaxies; Mamon (2000) recently concluded that the merger rate is 100fold higher in groups than in rich clusters. Groups are therefore the very best laboratory for studying the merger process, which is known to be the most effective in transforming galaxies morphologically (e.g. Toomre \& Toomre 1972).

- Groups contain a significant fraction of the hot gas in the universe: The total amount of hot gas in groups is comparable to that in clusters and therefore provides a significant contributor to the baryonic component of the universe (Fukugita et al. 1998). Groups provide 
an unprecedented opportunity to study the nature and origin of this important mass component and the intimate link it has with galaxies and their evolution.

- Star formation is suppressed on group scales: Large surveys, such as the 2dFGRS (Lewis et al. 2002) have revealed that star formation is suppressed at projected densities greater than one to two galaxies per square megaparsec. Such densities are associated with groups. The physical mechanism for this 'pre-processing' of galaxies in groups is currently not well understood (e.g. Fujita 2003; Bower \& Balogh 2003).

- A group is our local environment: Our Galaxy, the Milky Way, lives in a small loose group containing several dozen galaxies. A comprehensive understanding of galaxy groups in general will provide insight into our own Local Group.

Clusters arise from high-density pertubations. The early-type galaxies in clusters formed most of their stars at very early epochs, i.e. $z \geq 3$ (e.g. Bower, Lucey, \& Ellis 1992; Kelson et al. 1997; Jorgensen et al. 1999; van Dokkum et al. 2000; Terlevich \& Forbes 2002; Tanaka et al. 2005). Galaxy groups, on the other hand, are seeded by much lower density perturbations in a hierarchical Universe. They range from early-collapsed systems ('fossil groups') to overdensities only slightly greater than the 'field'. Star formation tends to be more extended in group galaxies (Terlevich \& Forbes 2002). Hence nearby groups offer the opportunity to study galaxy evolution and star formation histories in systems of different dynamical states. In addition, their proximity means that they are easily accessible with existing telescopes at different wavelengths.

Previous studies of galaxy groups have included those focussing on Hickson Compact Groups, e.g. the optical study by Hunsberger, Charlton, \& Zaritsky (1998), the X-ray study of Ponman et al. (1996), and the Hi survey by Verdes-Montenegro et al. (2001). On the slightly larger scale, we have poor (less than a dozen members), but relatively compact, groups have also attracted some attention (e.g. Tovmassian, Plionis, \& Andernach 2004; Plionis, Basilakos, \& Tovmassian 2004). Most studies of large loose groups have been restricted to small samples, perhaps due to the large areal coverage on the sky required. Wide area optical surveys have been conducted by Ferguson \& Sandage (1991) of five groups and Trentham \& Tully (2002) of four groups. Dedicated HI mapping of loose groups have been very few in number. Perhaps the largest is Maia, Willmer, \& da Costa (1998) who observed 73 (mostly loose) groups but without uniform coverage. The HIPASS Hi survey of the southern sky contains around one hundred HI-detected groups (Stevens 2005). X-ray studies have included similar numbers of loose groups (e.g. Mulchaey et al. 2000; Mahdavi et al. 2000; Helsdon et al. 2001).

However, most of these studies have tended to focus on a single wavelength. A notable exception is the optical and X-ray study of groups by Mulchaey \& Zabludoff
(1998) and Zabludoff \& Mulchaey (1998), who studied three compact and nine loose groups. Their groups were selected optically to be poor groups with less than five large galaxies. Nine of their groups were found to have extended X-ray emission; the remaining three were undetected in the X-ray. They found the nine groups with intragroup X-ray emission to be bound systems with many members (even the compact groups). These bound groups appear to share a common, massive halo, as opposed to possessing individual dark matter halos. This may explain why the galaxies in these groups have not yet all merged together. They also found bright elliptical galaxies at the centres of these groups, similar to those seen in clusters, and fractions of early-type galaxies approaching those in clusters. In contrast, the three groups undetected in X-rays were found to contain no early-type galaxies and have spiral galaxies at their centre. They also found that, in terms of their optical (velocity dispersion) and X-ray (X-ray luminosity and temperature) properties, groups are equivalent to scaleddown clusters.

The physical nature of groups, evolutionary connection between different types of groups, and the effect of the group environment on galaxies is not well understood. To fully understand any astrophysical problem it is important to adopt a multi-wavelength approach. This is particularly true for galaxy groups for which we expect large energetic and gas phase changes as a group evolves.

To overcome these limitations we created the Group Evolution Multiwavelength Study (GEMS) which is a survey of 60 nearby loose and compact galaxy groups. Here we describe the sample selection, the existing and planned data sets, and an overview of group properties. The GEMS collaboration involves researchers predominately from Swinburne University of Technology, Australia, and Birmingham University, UK, but includes others from many astronomical institutions world-wide. Further details can be obtained from our public web pages ${ }^{1}$.

\section{Sample Selection}

The GEMS sample is based on optically defined groups which have deep ROSAT X-ray data available. The presence of hot intragroup gas indicates that the group is real (rather than merely a projection on the sky and that it is a bound system). The X-ray luminosity gives a measure of the virialization of the group's potential well and hence its evolutionary state.

The groups of galaxies were initially selected from a master group catalogue consisting of 4320 opticallydefined groups (e.g. Garcia 1993; Tully 1987). This master group list was compared to the ROSAT satellite pointed observations, and all groups with a ROSAT PSPC pointing within 20 arcmin of the group position

\footnotetext{
${ }^{1}$ www.sr.bham.ac.uk/gems/
} 
were extracted. We further required that the ROSAT observation must be greater than $10^{4} \mathrm{~s}$ (sufficient for detailed X-ray analysis) and that the recession velocity of the group must lie between $1000 \mathrm{~km} \mathrm{~s}^{-1}<v_{\text {group }}$ $<3000 \mathrm{~km} \mathrm{~s}^{-1}$. This left a list of groups which should neither be too close so as to fill the ROSAT field of view nor too distant for detailed analysis. After excluding a small number of groups that are likely subclumps of the Hydra and Virgo clusters, this left 45 groups. To this we added 13 groups from Helsdon \& Ponman (2000), and two Hickson Compact Groups (HCG 4 and HCG 40) all with ROSAT PSPC imaging. Further details can be found in Osmond \& Ponman (2004).

So although not a statistically complete sample, the 60 groups have a large range in properties such as X-ray luminosity, dynamical state, velocity dispersion, richness, and constituent galaxy types, making them a representative sample of nearby galaxy groups and the largest multiwavelength sample to date. They cover the whole sky. The sample includes both loose and compact groups, thus we are in a position to test possible evolutionary connections between the two types of groups.

The distribution of the GEMS groups on the sky, in galactic coordinates, is shown in Figures 1 and 2. As can be seen in the figure, the GEMS groups were chosen to lie at $|l|>15^{\circ}$ from the Galactic plane.

\section{A Multiwavelength Data Set}

GEMS uses public catalogues/archive data as well as new dedicated observations. The main datasets are:

- X-ray imaging: All 60 groups have deep $\left(>10^{4} \mathrm{~s}\right)$ ROSAT PSPC images available. The ROSAT data, overlaid on DSS images, are shown in Figures 7-11. After taking into account point sources, 37 groups reveal extended $\mathrm{X}$-ray emission associated with an intragroup medium (called G), 15 groups have non extended X-ray emission that we associate with the galaxy halo $(\mathrm{H})$, and eight groups have X-ray emission that is less than $3 \sigma$ times the background and are classified as undetected (U). This diversity of X-ray properties can be seen in Figures 7-11. For further details see Osmond \& Ponman (2004). The ROSAT data are complemented by ever increasing coverage from the XMM-Newton and Chandra X-ray satellites (see Table 1), which have increased sensitivity and/or spatial resolution.

- HI mapping: We have carried out wide area $(5.5 \times$ $5.5 \mathrm{deg}^{2}$ ) Hi mapping with the Parkes radio telescope for 16 GEMS groups (see Table 1). Thus we sample out to radii of a few megaparsecs or several virial radii. The telescope was scanned across each group in a grid pattern for a total of 16-20 hours. The resulting velocity resolution is about $2 \mathrm{~km} \mathrm{~s}^{-1}$ (about ten times better than HIPASS) and a mass limit in the range $4-10 \times 10^{8} M_{\odot}$ (about two times better than HIPASS). The bandwidth is $8 \mathrm{MHz}$ with 1024 spectral channels. The final beam size is $15.5 \mathrm{arcmin}$. Source selection is quantified using fake sources injected into the datacubes and their recovery rate measured.

A small number of Parkes sources have also been observed on the Australia Telescope Compact Array. These are generally sources that are 'confused' in the large Parkes beam or new group members (see Kilborn et al. 2005b for details).

- Optical imaging: Wide-field imaging cameras have been used to image the central $0.5 \mathrm{deg}^{2}$ of 29 groups in $B, R, I$-filters. The data come from the $2.5-\mathrm{m}$ INT, Canary Islands (17 groups), the 2.2-m ESO/MPI telescope, Chile (eight groups), and the 3.9-m AAT, Australia (four groups). Seeing was around 1 arcsec. Galaxy selection was carried out using the SEXTRACTOR routine. The data reach to absolute magnitudes (assuming group membership) of $M_{B} \sim-13$. For further details see Miles et al. (2004).

- Near-infrared imaging: Image parameters and $J, H, K$ magnitudes are available for all GEMS group galaxies with $K<13.1$ from the 2MASS survey (Jarrett et al. 2000). These near-infrared magnitudes provide a good

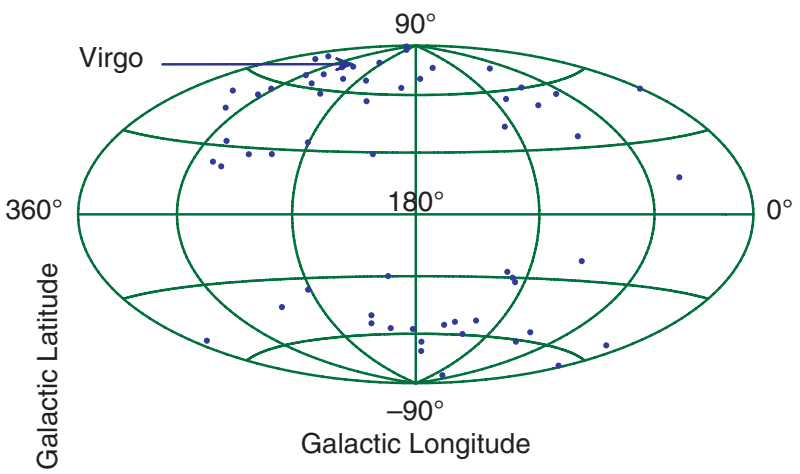

Figure 1 Distribution of the 60 GEMS groups on the sky in Galactic coordinates shown in an equal-area Aitoff projection. Lines of equal latitude and longitude are marked at intervals of $30^{\circ}$ and $60^{\circ}$ respectively. The position of the Virgo cluster is marked.

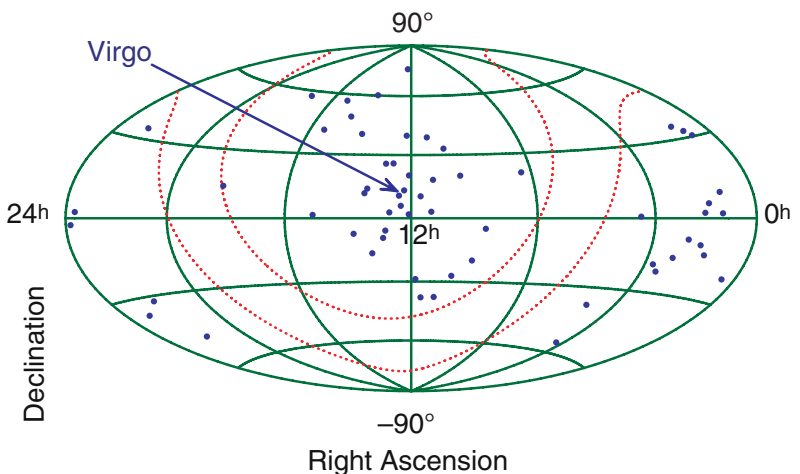

Figure 2 Distribution of the 60 GEMS groups on the sky in Equatorial coordinates shown in an equal-area Aitoff projection. Lines of equal RA and Dec. are marked at intervals of $60^{\circ}$ and $30^{\circ}$ respectively. The survey avoids groups with Galactic Latitude $|b|$ $<15^{\circ}$; these limits are shown as dotted lines. The position of the Virgo cluster is marked. 
Table 1. GEMS Group Properties

\begin{tabular}{|c|c|c|c|c|c|c|c|}
\hline Group name $\mathrm{A}^{\mathrm{A}}$ & $\begin{array}{c}\mathrm{RA}^{\mathrm{B}} \\
(\mathrm{J} 2000)\end{array}$ & $\begin{array}{l}\text { Dec. }^{\text {B }} \\
(\mathrm{J} 2000)\end{array}$ & $\begin{array}{c}\text { Distance }^{\mathrm{C}} \\
{[\mathrm{Mpc}]}\end{array}$ & $\begin{array}{l}r_{500}{ }^{\mathrm{D}} \\
{[\mathrm{Mpc}]}\end{array}$ & $\begin{array}{c}L_{X} \\
{\left[\operatorname{erg~s}^{-1}\right]}\end{array}$ & X-ray class ${ }^{\mathrm{E}}$ & Datasets $^{\mathrm{F}}$ \\
\hline HCG 4 & 003413.8 & -212621 & 115 & $0.36^{*}$ & $41.48 \pm 0.19$ & G & $\mathrm{C}, \mathrm{X}$ \\
\hline NGC 315 & 005748.9 & +302109 & 72 & 0.55 & $41.21 \pm 0.10$ & G & $\mathrm{C}$ \\
\hline NGC 383 & 010724.9 & +322445 & 73 & 0.69 & $43.07 \pm 0.01$ & $\mathrm{G}$ & $\mathrm{C}$ \\
\hline NGC 524 & 012447.8 & +093219 & 35 & 0.42 & $41.05 \pm 0.05$ & $\mathrm{H}$ & $\mathrm{P}, \mathrm{O}$ \\
\hline NGC 533 & 012531.3 & +014533 & 76 & 0.58 & $42.67 \pm 0.03$ & $\mathrm{G}$ & $\mathrm{C}, \mathrm{X}$ \\
\hline HCG 10 & 012540.4 & +344248 & 68 & 0.24 & $41.70 \pm 0.14$ & G & $\mathrm{O}$ \\
\hline NGC 720 & 015300.4 & -134418 & 23 & 0.40 & $41.20 \pm 0.02$ & $\mathrm{G}$ & $\mathrm{P}, \mathrm{O}, \mathrm{C}, \mathrm{X}$ \\
\hline NGC 741 & 015621.0 & +053744 & 79 & 0.62 & $42.44 \pm 0.06$ & G & C \\
\hline HCG 15 & 020737.5 & +021050 & 95 & 0.54 & $42.12 \pm 0.05$ & $\mathrm{G}$ & $\mathrm{X}$ \\
\hline HCG 16 & $\begin{array}{lll}02 & 09 & 24.7\end{array}$ & $\begin{array}{lll}-10 & 08 & 11\end{array}$ & 57 & 0.32 & $41.30 \pm 0.11$ & G & C,X \\
\hline NGC 1052 & 024104.8 & -081521 & 20 & 0.24 & $40.08 \pm 0.15$ & $\mathrm{H}$ & $\mathrm{P}, \mathrm{O}, \mathrm{C}, \mathrm{X}$ \\
\hline HCG 22 & 030331.0 & $\begin{array}{lll}-15 & 41 & 10\end{array}$ & 39 & 0.29 & $40.68 \pm 0.13$ & $\mathrm{G}$ & $\mathrm{O}$ \\
\hline NGC 1332 & 032617.1 & -212005 & 23 & 0.25 & $40.81 \pm 0.02$ & $\mathrm{H}$ & $\mathrm{P}, \mathrm{O}, \mathrm{C}$ \\
\hline NGC 1407 & 034011.8 & -183448 & 26 & 0.57 & $41.69 \pm 0.02$ & G & $\mathrm{P}, \mathrm{O}, \mathrm{C}$ \\
\hline NGC 1566 & 042000.6 & -545617 & 21 & 0.53 & $40.41 \pm 0.05$ & $\mathrm{H}$ & $\mathrm{P}, \mathrm{O}$ \\
\hline NGC 1587 & 043039.9 & +003943 & 55 & 0.55 & $41.18 \pm 0.09$ & $\mathrm{G}$ & $\mathrm{C}$ \\
\hline NGC 1808 & 050742.3 & -373046 & 17 & $0.32^{*}$ & $<40.10$ & $\mathrm{U}$ & $\mathrm{P}, \mathrm{O}, \mathrm{C}, \mathrm{X}$ \\
\hline NGC 2563 & 082035.7 & +210404 & 73 & 0.57 & $42.50 \pm 0.03$ & G & $\mathrm{O}, \mathrm{X}$ \\
\hline HCG 40 & 093854.5 & -045107 & 102 & $0.45^{*}$ & $<41.04$ & $\mathrm{U}$ & - \\
\hline HCG 42 & 100014.2 & -193803 & 64 & 0.48 & $41.99 \pm 0.02$ & $\mathrm{G}$ & $\mathrm{C}, \mathrm{X}$ \\
\hline NGC 3227 & 102330.6 & +195154 & 27 & $0.35^{*}$ & $41.23 \pm 0.05$ & $\mathrm{H}$ & $\mathrm{O}, \mathrm{C}, \mathrm{X}$ \\
\hline HCG 48 & 103749.5 & -270718 & 41 & $0.23^{*}$ & $41.09 \pm 0.04$ & $\mathrm{G}$ & - \\
\hline NGC 3396 & 104955.2 & +325927 & 31 & 0.36 & $40.53 \pm 0.08$ & $\mathrm{H}$ & $\mathrm{O}, \mathrm{C}, \mathrm{X}$ \\
\hline NGC 3557 & $\begin{array}{lll}11 & 09 & 57.4\end{array}$ & $\begin{array}{llll}-37 & 32 & 17\end{array}$ & 39 & 0.27 & $42.04 \pm 0.04$ & G & $\mathrm{P}, \mathrm{O}, \mathrm{C}$ \\
\hline NGC 3607 & 111654.7 & +180306 & 23 & 0.33 & $41.05 \pm 0.05$ & $\mathrm{G}$ & $\mathrm{O}, \mathrm{X}$ \\
\hline NGC 3640 & 112106.9 & +031406 & 29 & $0.35^{*}$ & $<40.37$ & $\mathrm{U}$ & $\mathrm{O}$ \\
\hline NGC 3665 & 112443.4 & +384544 & 37 & 0.38 & $41.11 \pm 0.08$ & $\mathrm{G}$ & $\mathrm{O}, \mathrm{C}, \mathrm{X}$ \\
\hline NGC 3783 & 113901.8 & -374419 & 36 & $0.25^{*}$ & $40.76 \pm 0.11$ & $\mathrm{G}$ & $\mathrm{P}, \mathrm{O}, \mathrm{C}, \mathrm{X}$ \\
\hline HCG 58 & 114223.7 & +101551 & 98 & $0.51^{*}$ & $<41.33$ & $\mathrm{U}$ & - \\
\hline NGC 3923 & 115102.1 & -284823 & 22 & 0.36 & $40.98 \pm 0.02$ & $\mathrm{H}$ & $\mathrm{P}, \mathrm{O}, \mathrm{C}, \mathrm{X}$ \\
\hline NGC 4065 & 120406.2 & +201406 & 106 & 0.62 & $42.64 \pm 0.05$ & G & X \\
\hline NGC 4073 & 120427.0 & +015348 & 96 & 0.69 & $43.41 \pm 0.02$ & G & $\mathrm{C}, \mathrm{X}$ \\
\hline NGC 4151 & 121032.6 & +392421 & 23 & $0.29^{*}$ & $<40.20$ & $\mathrm{U}$ & $\mathrm{O}, \mathrm{C}, \mathrm{X}$ \\
\hline NGC 4193 & 121353.6 & +131022 & 39 & $0.39^{*}$ & $40.63 \pm 0.08$ & $\mathrm{H}$ & - \\
\hline NGC 4261 & 121923.2 & +054931 & 41 & 0.64 & $41.92 \pm 0.03$ & G & $\mathrm{O}, \mathrm{C}, \mathrm{X}$ \\
\hline NGC 4325 & 122306.7 & +103716 & 117 & 0.51 & $43.15 \pm 0.01$ & G & $\mathrm{C}, \mathrm{X}$ \\
\hline NGC 4589 & 122145.0 & +751843 & 29 & 0.43 & $41.61 \pm 0.05$ & $\mathrm{G}$ & $\mathrm{X}$ \\
\hline NGC 4565 & 123620.8 & +255916 & 27 & 0.49 & $40.44 \pm 0.14$ & $\mathrm{H}$ & C,X \\
\hline NGC 4636 & 124250.4 & +024124 & 10 & 0.51 & $41.49 \pm 0.02$ & G & $\mathrm{P}, \mathrm{O}, \mathrm{C}, \mathrm{X}$ \\
\hline NGC 4697 & 124835.7 & -054803 & 20 & 0.39 & $41.01 \pm 0.02$ & $\mathrm{H}$ & O,C,X \\
\hline NGC 4725 & 125026.6 & +253006 & 25 & 0.43 & $40.63 \pm 0.06$ & $\mathrm{H}$ & $\mathrm{O}, \mathrm{C}, \mathrm{X}$ \\
\hline HCG 62 & 125305.8 & -091216 & 74 & 0.67 & $43.14 \pm 0.04$ & G & C,X \\
\hline NGC 5044 & 131524.0 & -162306 & 33 & 0.62 & $43.01 \pm 0.01$ & G & $\mathrm{P}, \mathrm{O}, \mathrm{C}, \mathrm{X}$ \\
\hline NGC 5129 & 132410.0 & +135836 & 108 & 0.51 & $42.33 \pm 0.04$ & G & X \\
\hline NGC 5171 & 132921.6 & +114407 & 107 & 0.58 & $42.38 \pm 0.06$ & G & $\mathrm{C}, \mathrm{X}$ \\
\hline HCG 67 & 134911.4 & -071328 & 115 & 0.46 & $42.02 \pm 0.07$ & $\mathrm{G}$ & - \\
\hline NGC 5322 & 134915.5 & +601128 & 35 & 0.42 & $40.71 \pm 0.10$ & $\mathrm{H}$ & $\mathrm{O}, \mathrm{X}$ \\
\hline HCG 68 & 135326.7 & +401659 & 41 & 0.43 & $41.52 \pm 0.04$ & $\mathrm{G}$ & $X$ \\
\hline NGC 5689 & 143452.0 & +483936 & 38 & $0.26^{*}$ & $<40.24$ & $\mathrm{U}$ & $X$ \\
\hline NGC 5846 & 150629.2 & +013621 & 30 & 0.48 & $41.90 \pm 0.02$ & $\mathrm{G}$ & $\mathrm{O}, \mathrm{C}, \mathrm{X}$ \\
\hline NGC 5907 & 151553.9 & +561946 & 17 & $0.24^{*}$ & $39.69 \pm 0.14$ & $\mathrm{H}$ & X \\
\hline NGC 5930 & 152607.9 & +414034 & 41 & 0.50 & $40.73 \pm 0.07$ & $\mathrm{H}$ & - \\
\hline
\end{tabular}


Table 1. Continued

\begin{tabular}{|c|c|c|c|c|c|c|c|}
\hline Group name $\mathrm{A}^{\mathrm{A}}$ & $\begin{array}{c}\mathrm{RA}^{\mathrm{B}} \\
(\mathrm{J} 2000)\end{array}$ & $\begin{array}{l}\text { Dec. }^{\text {B }} \\
\text { (J2000) }\end{array}$ & $\begin{array}{c}\text { Distance }^{\mathrm{C}} \\
{[\mathrm{Mpc}]}\end{array}$ & $\begin{array}{l}r_{500}{ }^{\mathrm{D}} \\
{[\mathrm{Mpc}]}\end{array}$ & $\begin{array}{c}L_{X} \\
{\left[\operatorname{erg~s} s^{-1}\right]}\end{array}$ & X-ray class ${ }^{\mathrm{E}}$ & Datasets $^{\mathrm{F}}$ \\
\hline NGC 6338 & 171522.9 & +572440 & 127 & $0.88^{*}$ & $43.51 \pm 0.02$ & $\mathrm{G}$ & $\mathrm{C}$ \\
\hline NGC 6574 & 181200.7 & +140244 & 35 & $0.16^{*}$ & $<40.81$ & $\mathrm{U}$ & 一 \\
\hline NGC 7144 & 215242.9 & -481516 & 27 & 0.30 & $40.33 \pm 0.13$ & $\mathrm{H}$ & $\mathrm{P}, \mathrm{O}$ \\
\hline HCG 90 & 220208.4 & -315930 & 36 & 0.38 & $41.49 \pm 0.05$ & G & $\mathrm{P}, \mathrm{O}, \mathrm{C}, \mathrm{X}$ \\
\hline HCG 92 & 223558.4 & +335757 & 88 & 0.47 & $41.99 \pm 0.04$ & $\mathrm{G}$ & C,X \\
\hline IC 1459 & 225710.6 & -362744 & 26 & 0.35 & $41.28 \pm 0.04$ & G & $\mathrm{P}, \mathrm{O}, \mathrm{C}, \mathrm{X}$ \\
\hline NGC 7714 & 233614.1 & +020919 & 39 & $0.22^{*}$ & $<40.03$ & $\mathrm{U}$ & $\mathrm{P}, \mathrm{X}$ \\
\hline HCG 97 & 234722.9 & -021802 & 92 & 0.51 & $42.37 \pm 0.05$ & $\mathrm{G}$ & $\mathrm{X}$ \\
\hline
\end{tabular}

${ }^{\text {A }}$ Generally the brightest central galaxy or HCG number. ${ }^{\mathrm{B}}$ Position of centrally located galaxy or optical group catalog. ${ }^{\mathrm{C}}$ Distance from Virgo-infall corrected velocity and assuming $H_{0}=70 \mathrm{~km} \mathrm{~s}^{-1} \mathrm{Mpc}^{-1}$. ${ }^{\mathrm{D}}$ Radius at 500 times the critical density $\left(^{*}=\right.$ uncertain radius). ${ }^{\mathrm{E}} \mathrm{G}=$ intragroup emission, $\mathrm{H}=$ galaxy halo emission, $\mathrm{U}=$ undetected in $\mathrm{X}$-rays. ${ }^{\mathrm{F}} \mathrm{O}=$ optical imaging, $\mathrm{P}=\mathrm{Parkes} \mathrm{HI}$ imaging, $\mathrm{C}=$ Chandra $\mathrm{X}$-ray mapping, $\mathrm{X}=\mathrm{XMM}$-Newton $\mathrm{X}$-ray mapping.

(photometric) tracer of galaxy mass, and are used in GEMS to calculate luminosity-weighted group centroid positions and to analyze group properties (see Brough et al. 2005 for details).

- Recession velocities: Confirmed group membership requires a recession velocity from either optical spectra or $\mathrm{H}_{\mathrm{I}}$ observations. We complement the existing databases (such as NED and Hyperleda) with our own new Hi velocities and those from the $6 \mathrm{dFGS}$ (Jones et al. 2004) for some southern groups. More velocities will become available after the third and final 6dFGS data release. The SDSS survey will confirm group membership for many northern groups. Such velocities are being used to derive group properties such as velocity dispersions and hence probe group dynamics (e.g. Brough et al. 2005) and can be used to test whether HCGs are merely the dense cores of larger loose groups.

\section{Mock Catalogues}

Sample selection is one aspect of the study of galaxy groups that has significantly hindered progress. A large, well-selected sample has simply been lacking. This is partly due to the difficulty of defining galaxy groups in an objective way from galaxy positions alone (see Eke et al. 2004) combined with the difficulty of detecting X-ray emission from groups of $\leq 10^{13} h^{-1} M_{\odot}$ (e.g. Osmond \& Ponman 2004). To circumvent these problems and test our group selection procedure we use modern simulations.

\subsection{Dark Matter and Stellar Content}

Our mock Universe is based on the $500 \mathrm{~h}^{-1} \mathrm{Mpc}$ volume of the Millennium simulation (Springel et al. 2005). With over $10^{10}$ particles each of mass $8.6 \times 10^{8} h^{-1} M_{\odot}$ this model resolves halos containing typical galaxy groups with over $10^{4}$ particles. There are over $5 \times 10^{4}$ halos of group or larger size within the simulation volume, allowing detailed statistics of the halo properties to be extracted.
Within the framework of the Millennium simulation we have access to the mock catalogues of both Croton et al. (2005) and Bower et al. (2005). Both these mock catalogues include AGN feedback and successfully reproduce the break in the galaxy luminosity function.

\subsection{Gas Properties}

The $500 h^{-1} \mathrm{Mpc}$ volume of the Millennium simulation has also been modelled at lower resolution but including hydrodynamics. Although this lower resolution is insufficient for a full treatment of the gaseous phase of galaxies it is sufficient to resolve the hot halos and produce reliable $\mathrm{X}$-ray luminosities and gas temperatures. We have two runs, one without gas cooling (which produces halos that are far too bright in X-rays) and a run with preheating that has been tuned to reproduce both the observed X-ray luminosity-temperature relation and the mass-temperature relation. These models accurately reproduce the halo distribution of the higher resolution Millennium simulation.

With this set of models we can inter-compare galaxy, dark matter, and gas properties for a volumelimited sample of more than $5 \times 10^{4}$ halos. We can also examine the evolution of any desired halo or set of halos, as both the dark matter and galaxy catalogues have 64 output redshifts, a number increased further still to 160 in the gas models.

As an example of what is available, we have extracted the 1D dark matter velocity dispersion within the virial radius of all the haloes with $M \geq 10^{13} h^{-1} M_{\odot}$. In Figure 3 we compare this to the $3 \mathrm{D} / \sqrt{3}$ (black points) and $1 \mathrm{D}$ (red points) velocity dispersion for all galaxies with $M_{B}<-16$ within these halos. At low velocity dispersion (small groups), the 1D line-of-sight measurement becomes a less reliable indicator of the true group velocity dispersion.

Figure 4 displays the same information but this time only the median, 10th, and 90th percentile values are shown. For small haloes there is a slight tendency for the 


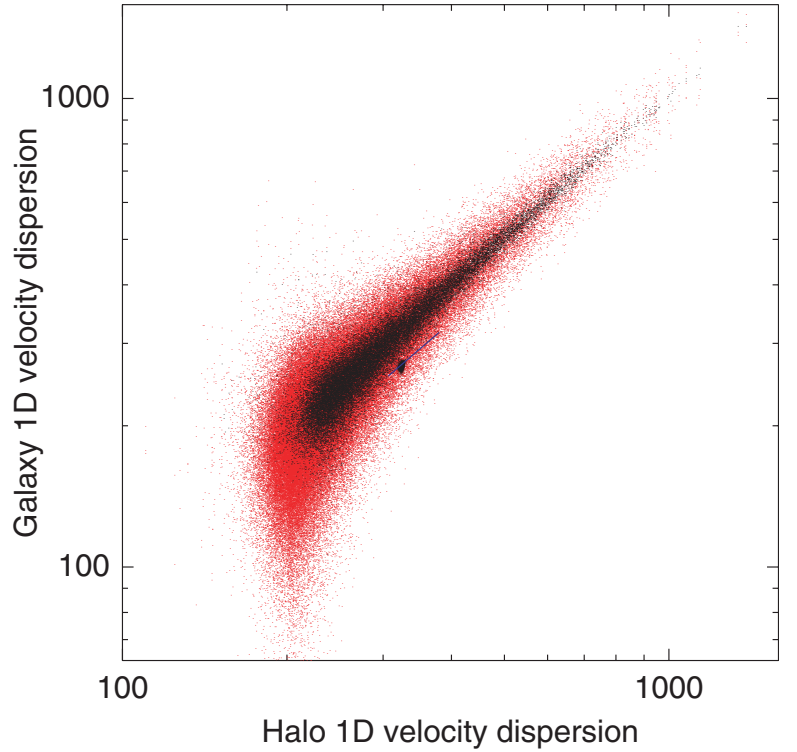

Figure 3 Velocity dispersions from mock catalogues. The plot shows the measured $3 \mathrm{D} / \sqrt{3}$ (black points) and $1 \mathrm{D}$ (red points, in three orthogonal directions for each group/cluster) velocity dispersion versus the true dark matter velocity dispersion of the halo for all galaxies $M_{B}<$ -16 within the virial radius of group-sized $\left(M \geq 10^{13} h^{-1} M_{\odot}\right)$ and larger halos. The solid blue line shows the one-to-one relation. The plot contains data for over $5 \times 10^{4}$ halos. At low velocity dispersion (small groups), the 1D line-of-sight measurement becomes a less reliable indicator of the true group velocity dispersion and the systems themselves may not be true groups.

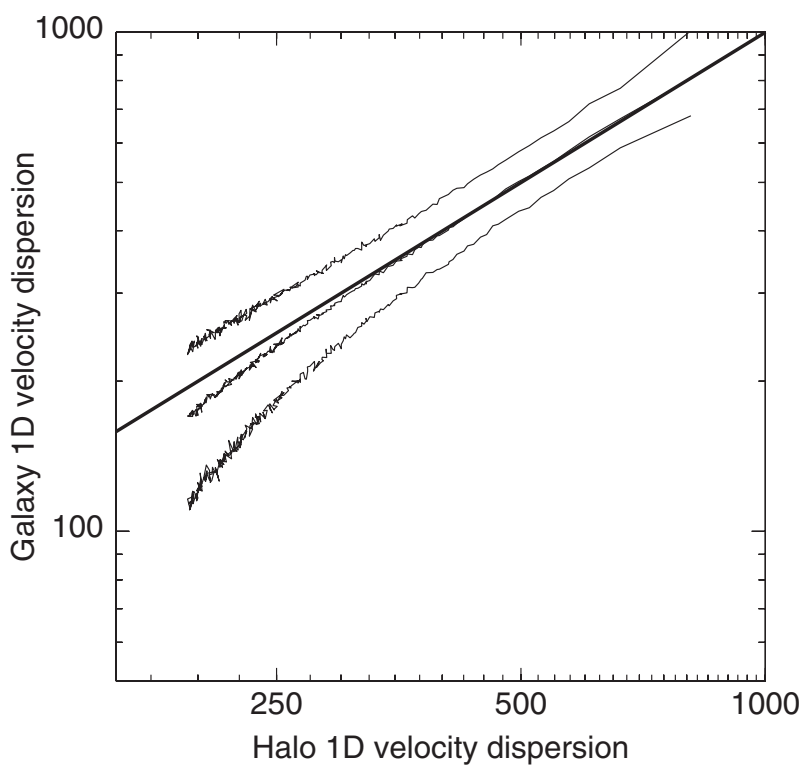

Figure 4 The median velocity dispersions from the mock catalogues. Data from Figure 3 is reproduced as the black and red lines, which show the mean, 10th percentile, and 90th percentile for bins of 200 halos of the specified dark matter velocity dispersion. For small haloes there is a slight tendency for the galaxies to have a lower than expected velocity dispersion. The solid blue line shows the one-to-one relation. The plot shows that for low velocity dispersion groups the dispersion is not a good indicator of the true group size or mass.

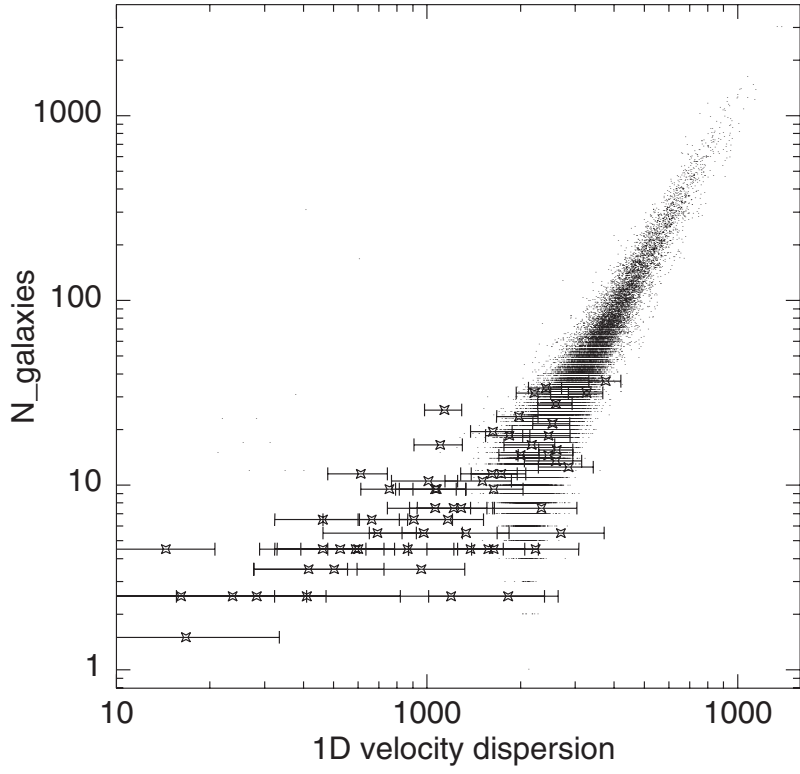

Figure 5 The number of galaxies within each halo. The black points show the number of $M_{B}<-16.32$ simulated galaxies within a cylinder of radius $r_{500}$ as a function of the 1D velocity dispersion of the halo in the catalogues of Croton et al. (2005). The red points with error bars are the GEMS group sample of Osmond \& Ponman (2004), also limited at $M_{B}<-16.32$.

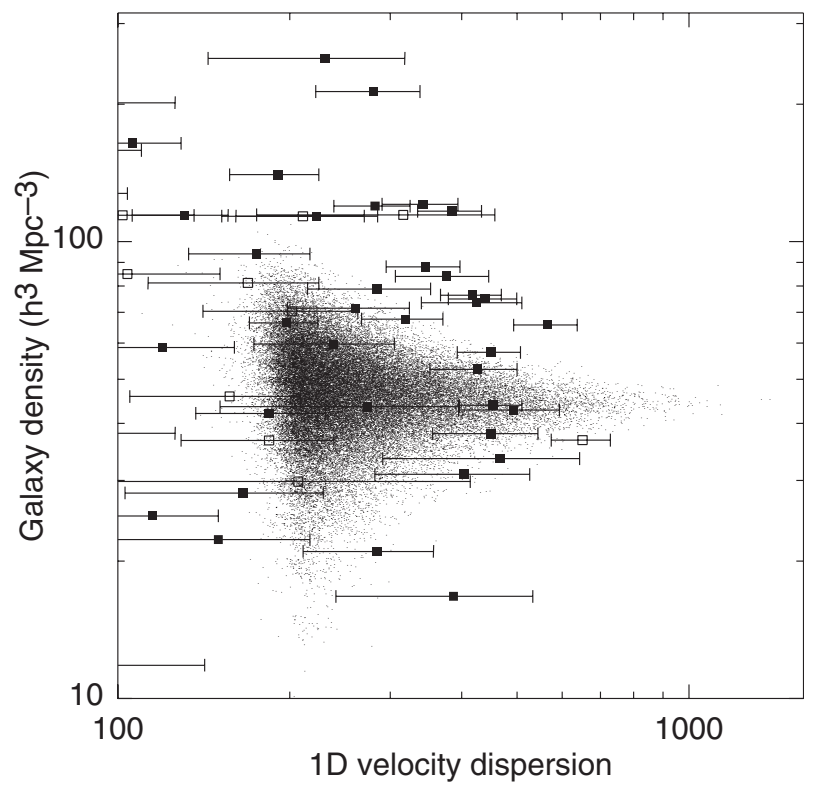

Figure 6 The galaxy density within each halo. The black points show the measured galaxy density for $M_{B}<-16.32$ simulated galaxies, where we have taken the volume of a sphere of radius $r_{500}$ but the number of galaxies counted within a cylinder of this radius (to match the observational method). We see increased scatter in galaxy density at low velocity dispersion groups, which are only partially resolved in our simulations. The red squares with errorbars are the GEMS groups of Osmond \& Ponman (2004); open squares indicating the groups with unreliable sizes. 

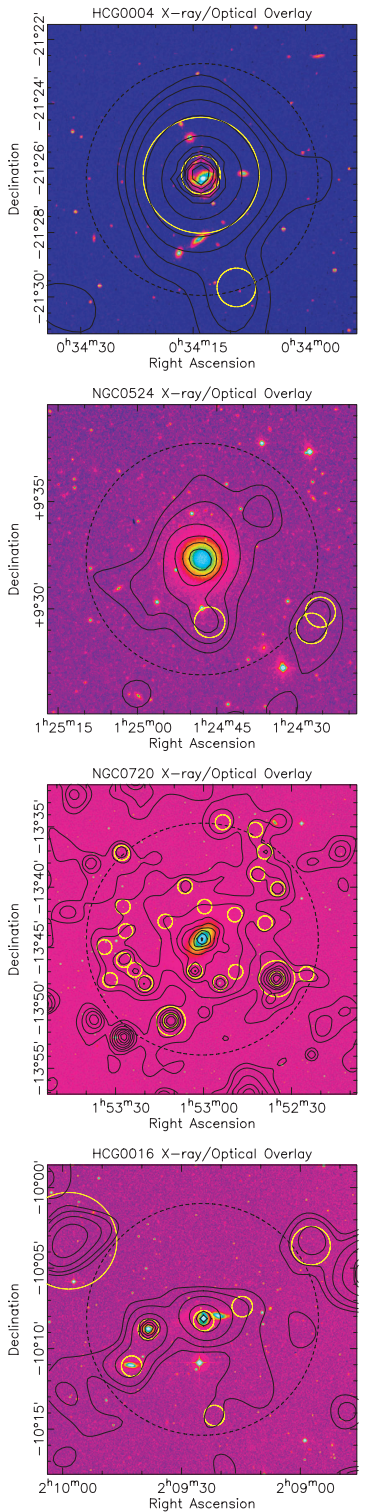
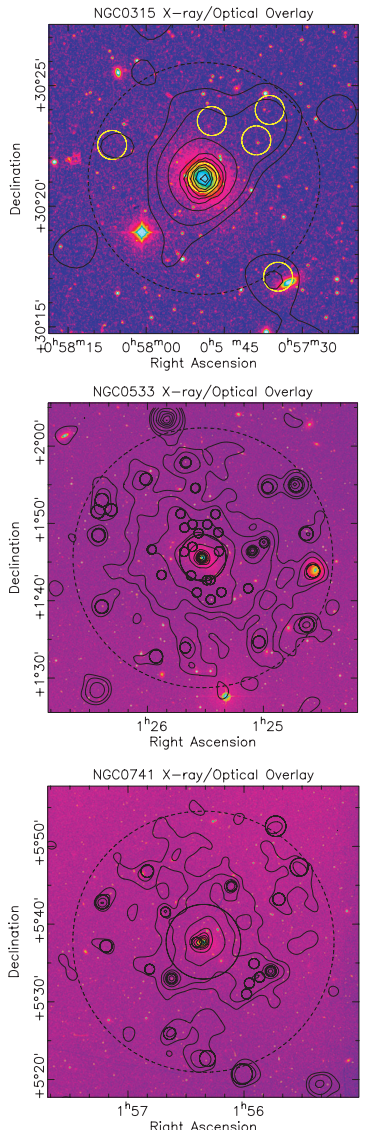

$1^{h_{57}}{ }_{\text {Right Ascension }}^{1^{h_{5}}}$

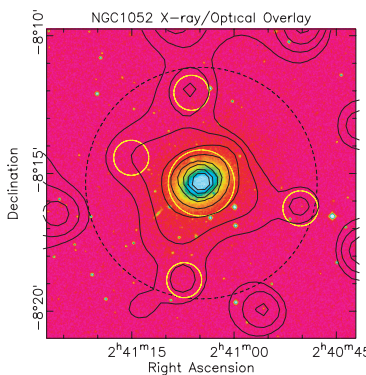

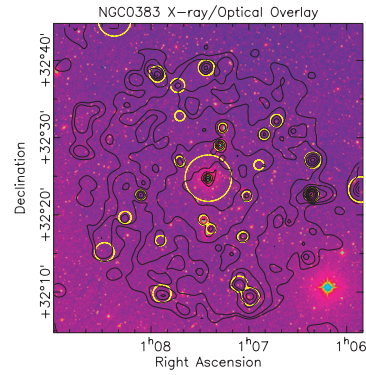

Right Ascension

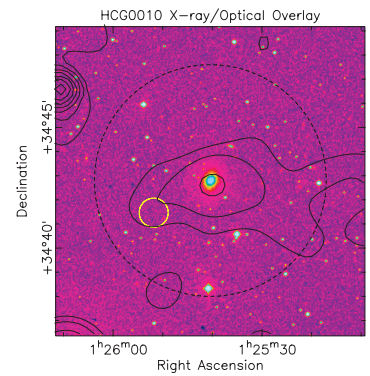

HCG0015 X-ray/0ptical Overloy
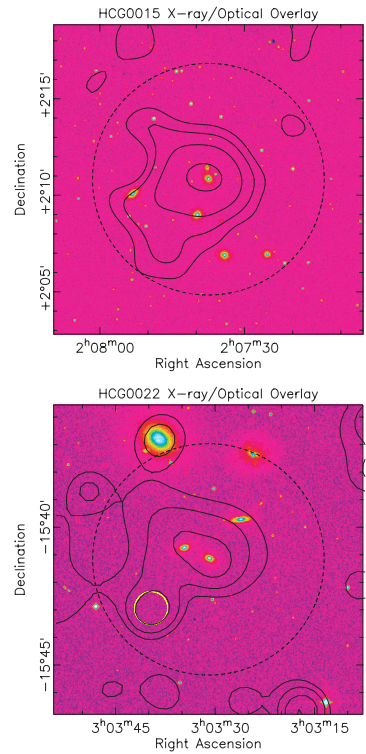

Figure 7 The GEMS groups. ROSAT X-ray contours overlaid on optical DSS images. The dashed line represents the 'cut-off radius' at which the X-ray emission reaches the background level. The small yellow circles indicate point sources. Large yellow circles indicate that point sources dominate that region.

galaxies to have a lower than expected velocity dispersion, due to the fact that the semi-analytic galaxy is always the most-bound particle of its parent halo. No correction is made for the small numbers of galaxies or the fact that the central galaxy is assumed to be at rest with respect to the group. Due to the fact that we have over $5 \times 10^{4}$ clusters and $15 \times 10^{4}$ independent measures of the velocity dispersion our sample contains several hundred groups with derived velocity dispersions below $100 \mathrm{~km} \mathrm{~s}^{-1}$. If we solely used the galaxy velocity dispersion to derive the size of these groups we would arrive at far too small a number. The sharp drop seen in Figure 3 is mainly driven because we have deliberately chosen to cut off our sample at $10^{13} h^{-1} M_{\odot}$ so that there are suddenly no smaller groups in our sample. Something like this effect also occurs observationally: Below a certain size, groups are not identified because they only contain a single galaxy. Slightly above this threshold the recovered velocity dispersion is, on average, $6 \%$ too high - this is simply because there are more smaller groups than larger ones, so more groups scatter up than down. In summary, velocity dispersion measures for low mass groups become increasingly unreliable with a tendency to underestimate the group velocity dispersion (and hence underestimate group mass).

\subsection{Galaxy Counts}

The number of galaxies above a specified brightness (in this case $M_{B}<-16.32$ ) is well correlated with the halo velocity dispersion (Figure 5). The GEMS group sample of Osmond \& Ponman (2004) spans the model results and extends them to lower velocity dispersions. Many of the lower mass GEMS groups may not be virialized systems. The galaxy 

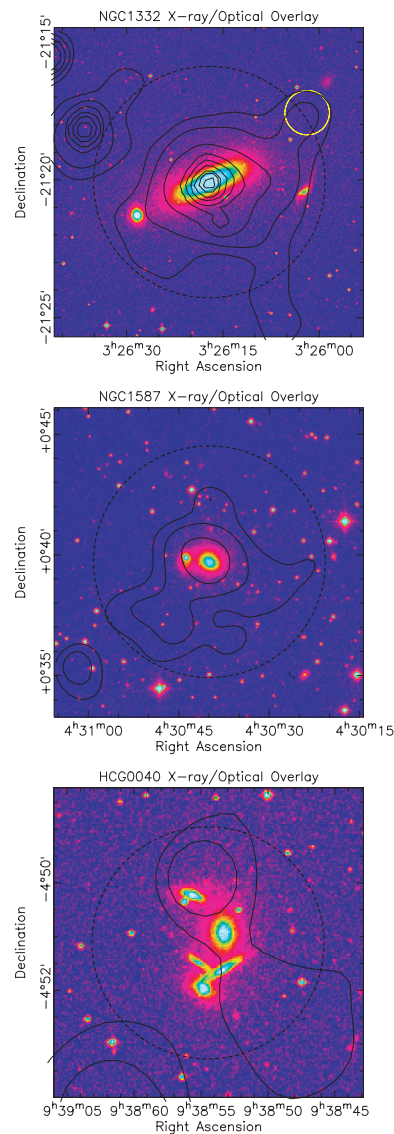

HCG0048 X-roy/Optical Overloy

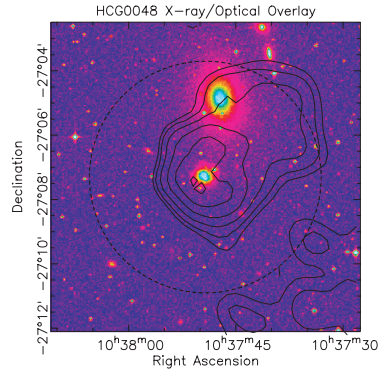

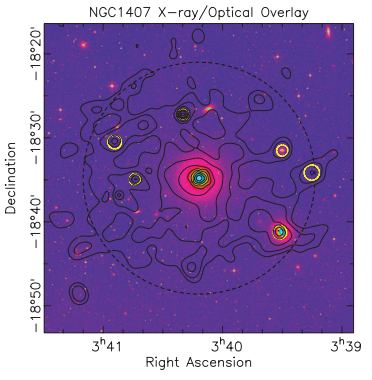

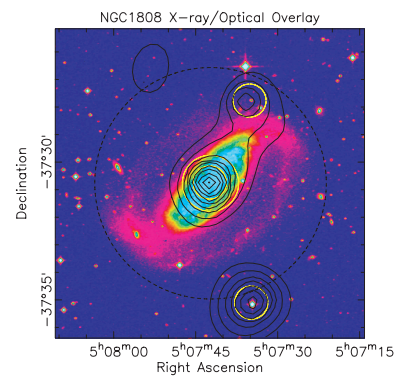

Hccose $x$-roy/0pticat Overloy

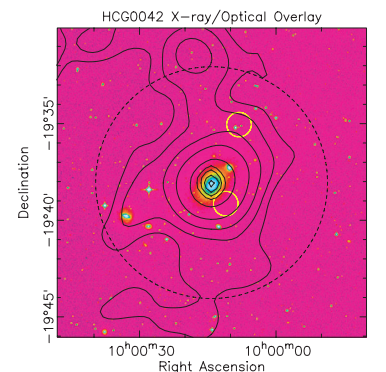

NGC3396 X-ray/Optical Overlay

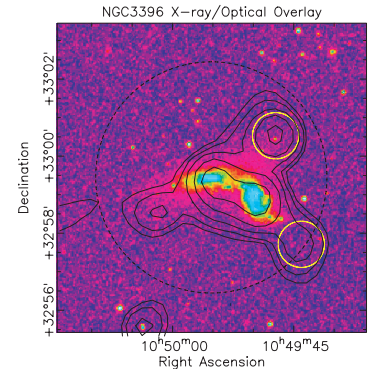

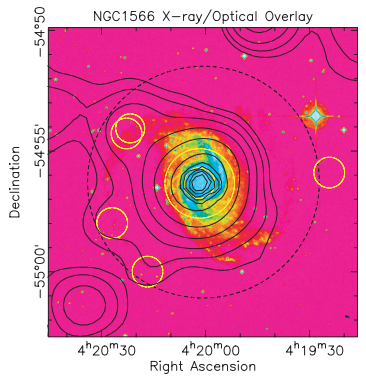
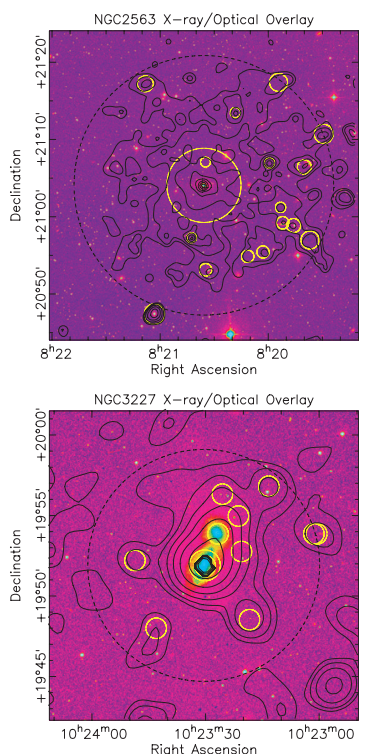

NGC3557 X-ray/Optical Overloy

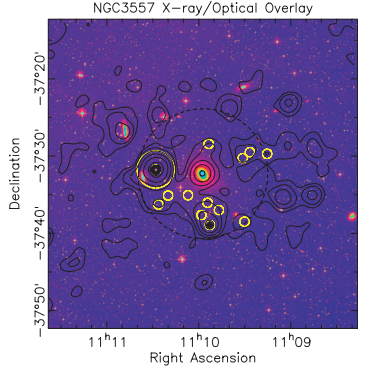

Figure 8 The GEMS groups. ROSAT X-ray contours overlaid on optical DSS images. The dashed line represents the 'cut-off radius' at which the X-ray emission reaches the background level. The small yellow circles indicate point sources. Large yellow circles indicate that point sources dominate that region.

density (for galaxies brighter than $M_{B}<-16.32$ ) is stable at a value of around 45 galaxies per $h^{-1} \mathrm{Mpc}^{3}$ although the scatter increases dramatically as the velocity dispersion of the halo drops (Figure 6). Again the Osmond \& Ponman groups span the model range, with no trend for a large systematic increase in galaxy density as the velocity dispersion is lowered; such a trend would be a clear indication that the size of the groups (and hence masses) had been systematically underestimated. This is not a problem for the GEMS groups, as discussed in Osmond \& Ponman (2004), section 6 .

\section{Group Properties}

We summarize some basic properties of the 60 GEMS groups in Table 1 . The group name is generally the brightest central galaxy or Hickson Compact Group number. The position corresponds to the centrally located galaxy or that listed in the optical group catalog (see Osmond \& Ponman 2004). The distance is calculated from a Virgo-infall corrected velocity and assuming $H_{0}=70 \mathrm{~km} \mathrm{~s}^{-1} \mathrm{Mpc}^{-1}$ (this Hubble constant is used in all GEMS papers). The $r_{500}$ radius is a measure of the extent of each group. It is defined as the radius for which the density is 500 times the critical density of the Universe. For groups, this quantity is more reliably obtained than the commonly used $r_{200}$ or virial radius $\left(r_{500} \approx 2 / 3 r_{200}\right)$ in clusters. It is calculated based on the simulations of Evrard et al. (1996) using the measured $\mathrm{X}$-ray temperature, $T_{\mathrm{X}}$ (for those groups without $\mathrm{X}$-ray temperatures, it is calculated from the summed $B$-band luminosity $\left(L_{B}\right)$ of all galaxies in the group). The $r_{500}$ values listed here have been updated from Osmond \& Ponman 

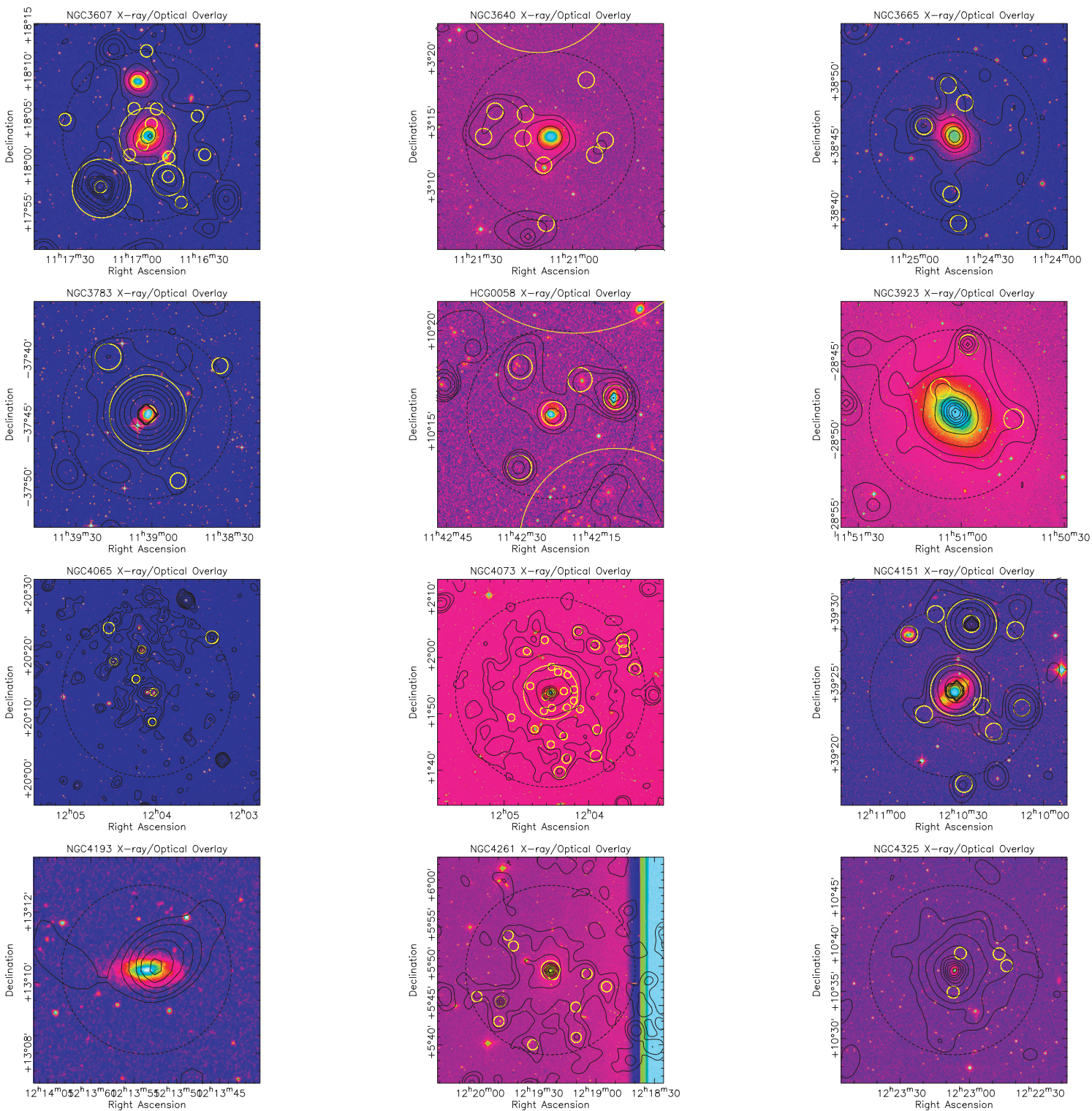

Figure 9 The GEMS groups. ROSAT X-ray contours overlaid on optical DSS images. The dashed line represents the 'cut-off radius' at which the X-ray emission reaches the background level. The small yellow circles indicate point sources. Large yellow circles indicate that point sources dominate that region.

(2004) so that for groups with galaxy halo emission the radius is now calculated based on $L_{B}$ (rather than $T_{\mathrm{X}}$ ). The measured X-ray luminosity and upper limits are from Osmond \& Ponman (2004). Whether this X-ray emission comes from intragroup emission $(\mathrm{G})$, galaxy halo emission $(\mathrm{H})$, or is undetected in X-rays (U) is listed.

\section{GEMS Publications}

Since the first presentation at the European National Astronomy meeting (McKay et al. 2002), there have been several GEMS publications:

- Khosroshahi et al. (2004): An analysis of galaxy surface brightness profiles in 16 groups, which found galaxies in X-ray luminous groups to be in general larger and more luminous than their counterparts in low X-ray groups.
- Osmond \& Ponman (2004): An analysis of the X-ray properties of the entire sample. X-ray luminosities to a uniform group scale radius are derived. More recent fits to the $L_{X}$ scaling relations (Ponman et al. 2006) indicate that the X-ray properties of groups are consistent with being scaled-down clusters. Various group optical properties are found to be correlated to X-ray properties (e.g. the spiral fraction with IGM X-ray temperature).

- McKay et al. (2004): This paper reports the discovery of two new galaxies, from Parkes Hi mapping, in the NGC 1052 and NGC 5044 groups. The two galaxies have $\mathrm{H}_{\mathrm{r}}$ masses of $0.5-1.0 \times 10^{9} M_{\odot}$ and faint optical counterparts.

- Miles et al. (2004): Luminosity functions, in the $B$ - and $R$-bands, are presented for the central regions of 25 groups. A strong dip in the $\mathrm{LF}$ is seen around $M_{B}=-17$ in the low X-ray groups. This may be interpreted as a 

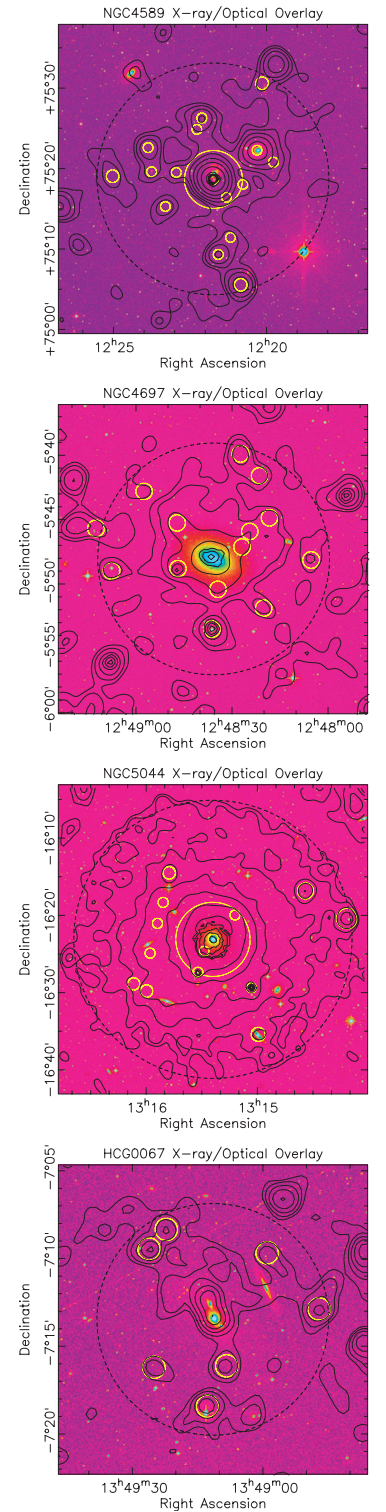

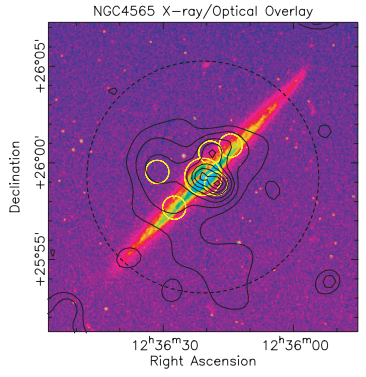

4725 X-ray/Optical Overlay
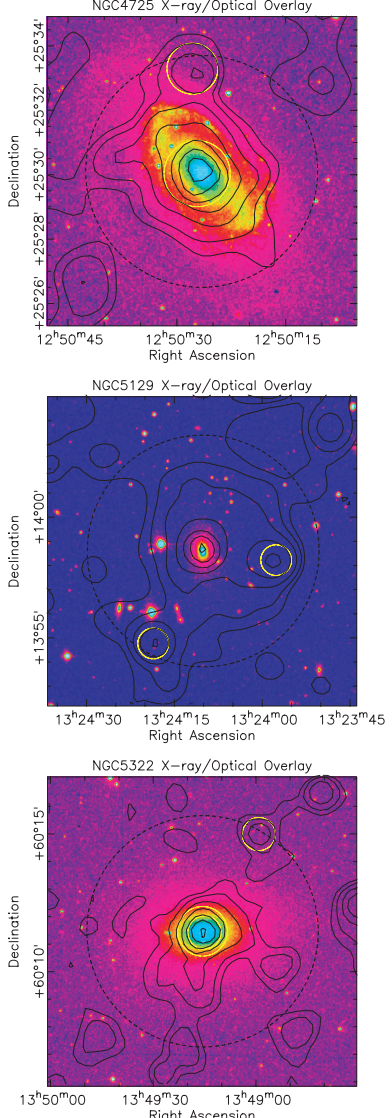
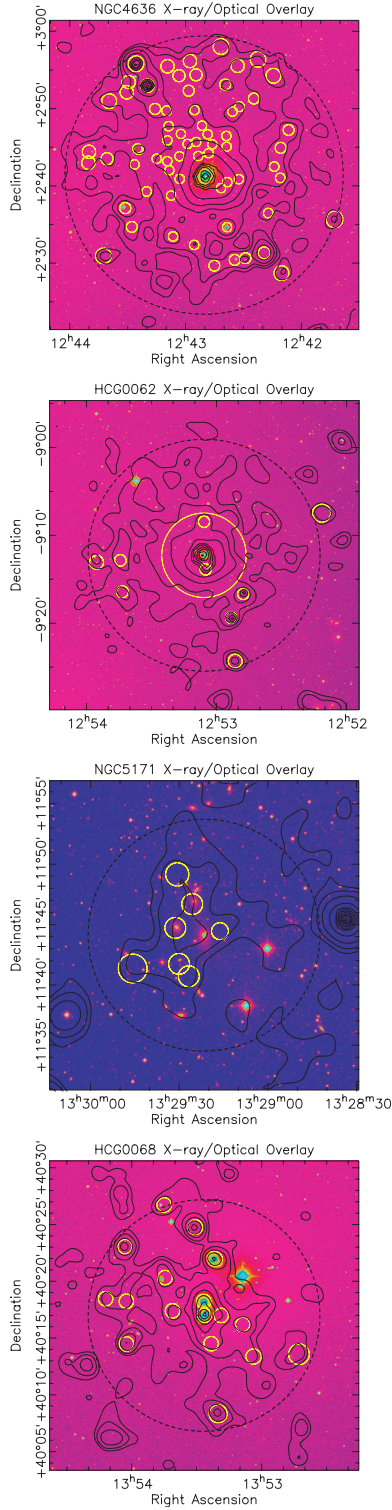

Figure 10 The GEMS groups. ROSAT X-ray contours overlaid on optical DSS images. The dashed line represents the 'cut-off radius' at which the X-ray emission reaches the background level. The small yellow circles indicate point sources. Large yellow circles indicate that point sources dominate that region.

signature of merging at intermediate galaxy mass scales in pre-virialized, collapsing groups.

- Kilborn et al. (2005a): This paper presents wide area Parkes Hi mapping of the low X-ray emission group NGC 1566. Of the $13 \mathrm{HI}$-detected galaxies, two were not known previously to be group members. Several galaxies appear be HI-deficient, the cause of which is probably tidal interactions rather than ram pressure stripping.

- Brough et al. (2005): The dynamics of the GEMS groups NGC 1407 and NGC 1332 are studied, along with the Eridanus cloud. It is suggested these groups form a bound 'supergroup' of $7 \times 10^{13} M \odot$ that is due to merge into a cluster.

- Kilborn et al. (2005b): This paper presents wide area Parkes HI mapping of the NGC 3783 group, which has some evidence for a weak IGM. We find one
HI 'cloud' (i.e. detected HI with no optical counterpart to faint levels) of mass of about $10^{9} M_{\odot}$. This cloud is likely to have a tidal origin.

\section{Summary and Future Work}

Groups are the preferred environment of galaxies for more than half of the age of the Universe. The GEMS project is an attempt to better quantify and understand the processes operating in nearby galaxy groups, building on the work of Zabludoff, Mulchaey, and others from the 1990s. Our sample of 60 groups covers a range of group dynamical states. Our multi-wavelength dataset and mock catalogues allow us to define the properties of these groups and better understand the physical processes operating. Our existing, and future, GEMS publications will provide a useful 'local benchmark' for groups found at moderate to high redshift 

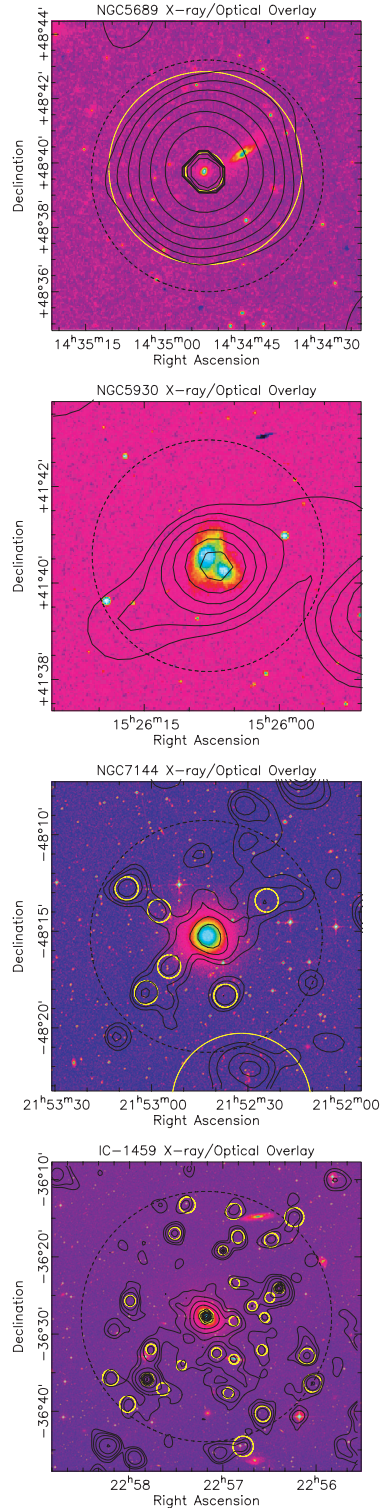
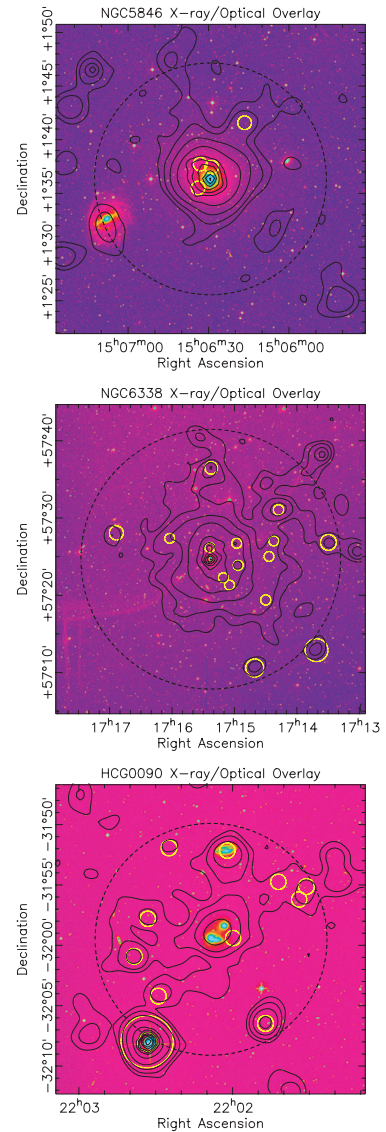

NGC7714 X-ray/Optical Overlay

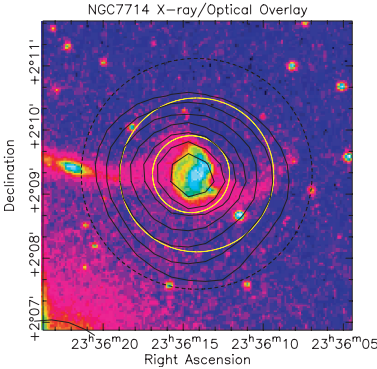

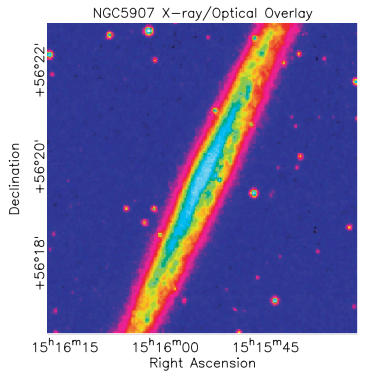
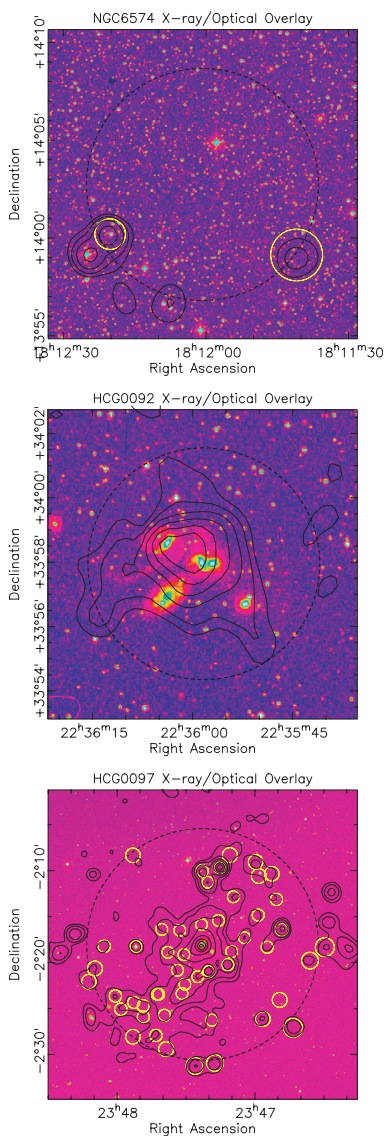

Figure 11 The GEMS groups. ROSAT X-ray contours overlaid on optical DSS images. The dashed line represents the 'cut-off radius' at which the X-ray emission reaches the background level. The small yellow circles indicate point sources. Large yellow circles indicate that point sources dominate that region.

in galaxy surveys such as the 2dFGRS, SDSS, DEEP2, and 6dFGS.

Future work includes measuring the $K$-band galaxy luminosity function, a dynamical analysis of galaxy motions, high spatial resolution Hi follow-up of selected galaxies, examination of the star formation rates through $\mathrm{H} \alpha$ fluxes, and the origin of brightest group galaxies.

\section{Acknowledgments}

Thanks to the ARC for funding aspects of this research and the telescope support staff at various observatories who helped collect the GEMS datasets. We thank the following for their help with aspects of GEMS: D. Barnes, P. Goudfrooij, S. Helsdon, H. Khosroshahi, B. Koribalski, N. McKay, P. Nulsen, and R. Proctor.

\section{Accessory Materials}

High-resolution versions of the images in Figures 7-11 are available from the author or, until March 2011, Publications of the Astronomical Society of Australia.

\section{References}

Bekki, K. 1999 ApJL, 510, 15

Bower, R., Lucey, J., \& Ellis, R. 1992, MNRAS, 254, 601

Bower, R., \& Balogh, M. 2003, RMxAC, 17, 220

Bower, R., et al. 2005, astro-ph/0511338

Brough, S., et al. 2005, submitted

Croton, D., et al. 2005, astro-ph/0508046

Eke, V., et al. 2004, MNRAS, 348, 866

Evrard, A., Metzler, C., \& Navarro, J. 1996, ApJ, 469, 494

Ferguson, H., \& Sandage, A. 1991, AJ, 101, 765

Fukugita, M., Hogan, C., \& Peebles, J. 1998, ApJ, 503, 518

Fujita, Y. 2003, astro-ph/0311193 
Garcia, A. 1993, A\&AS, 100, 47

Helsdon, S., \& Ponman, T. 2000, MNRAS, 319, 933

Helsdon, S., Ponman, T., O’Sullivan, E., \& Forbes, D. 2001, MNRAS, 325, 693

Hunsberger, S., Charlton, J., \& Zaritsky, D. 1998, ApJ, 505, 536 Jarrett, T., Chester, T., Cutri, R., Schneider, S., Skrutskie, M., \& Huchra, J. 2000, AJ, 119, 2498

Jones, D., et al. 2004, MNRAS, 355, 747

Jorgensen, I., et al. 1999, MNRAS, 308, 833

Kelson, D., et al. 1997, ApJL, 478, 13

Kilborn et al. 2005a, MNRAS, 356, 77

Kilborn et al. 2005b, MNRAS, submitted

Khosroshahi et al. 2004, MNRAS, 349, 527

Lewis, I., et al. 2002, MNRAS, 334, 673

Mahdavi, A., Bohringer, H., Geller, M., \& Ramella, M. 2000, ApJ, 534, 114

Maia, M. A. G., Willmer, C. N. A., \& da Costa, L. N. 1998, AJ, 115, 49

Mamon, G. 2000, in 'Dynamics of Galaxies: from the Early Universe to the Present' (Eds Combes, F., Mamon, G. A., \& Charmandaris, V.) ASP Conference Series, p. 377

McKay, N., Mundell, C., Brough, S., Forbes, D., \& Barnes, D. 2002, in 'Galaxy Evolution in Groups and Clusters' (Eds Lobo, C., Serote Roos, M., \& Biviano, A.) (Amsterdam: Kluwer), astro-ph/0212238

McKay et al. 2004, MNRAS, 352, 1121

Meurer, G. 2003, astro-ph/0311184
Miles et al. 2004, MNRAS, 355, 785

Moss, C. \& Whittle, M. 2000, MNRAS, 317, 667

Mulchaey, J. 2000, ARAA, 38, 289

Mulchaey, J., \& Zabludoff, A. 1998, ApJ, 496, 73

Osmond, J., \& Ponman, T. 2004, MNRAS, 350, 1511

Plionis, M., Basilakos, S., \& Tovmassian, H. 2004, MNRAS, 352, 1323

Ponman, T., Bourner, P., Ebeling, H., \& Bohringer, H. 1996, MNRAS, 283, 690

Ponman et al. 2006, in preparation

Solanes, J.M., Salvador-Solé, E., \& González-Casado, G. 1999, A\&A, 343, 733

Springel, V., et al. 2005, Natur, 435, 629

Stevens, J. 2005, PhD Thesis (Melbourne: University of Melbourne)

Tanaka, M., et al. 2005, MNRAS, 362, 268

Terlevich, A., \& Forbes, D. 2002, MNRAS, 330, 547

Toomre, A., \& Toomre, J. 1972, ApJ, 178, 623

Tovmassian, H., Plionis, M., \& Andernach, H. 2004, ApJL, 617, 111

Trentham, N., \& Tully, R. 2002, MNRAS, 335, 712

Tully, R. B. 1987, ApJ, 321, 280

van Dokkum, P., et al. 2000, ApJ, 541, 95

Verdes-Montenegro, L., Yun, M., Williams, B., Huchtmeier, W., Del Olmo, A., \& Perea, J. 2001, A\&A, 377, 812

Zabludoff, A., \& Mulchaey, J. 1998, ApJ, 496, 39 